\title{
Therapeutisches Ethos und Vertraulichkeit im Spannungsfeld der Kulturen
}

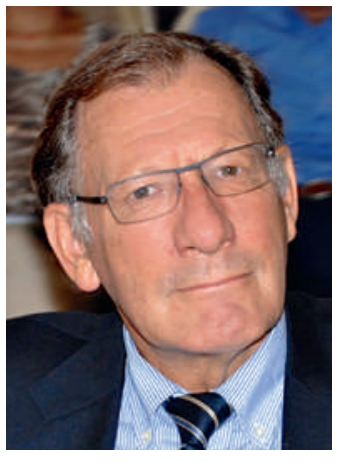

Jean Martin

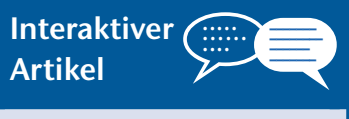

Wollen Sie diesen Artikel kommentieren? Nutzen Sie dafür die Kommentarfunktion in der OnlineVersion oder sehen Sie nach, was Ihre Kolleginnen und Kollegen bereits geschrieben haben: www.saez.ch/ aktuelle-ausgabe/ interaktive-beitraege/
Nicht selten äussere ich Bedenken wegen eines gewissen westlichen Kulturimperialismus, der auf mangelndem Verständnis fremder Wertesysteme beruht. Dennoch steht ausser Frage, dass gewisse Praktiken strikt abzulehnen sind - und zwar in den Ländern, sofern dies machbar ist, in jedem Fall aber bei uns. Dies gilt für «Ehrenmorde», wie sie im Nahen Osten oder auch weiterhin in Asien noch üblich sind. Sie zu verhüten, erfordert ein aufmerksames Auge der Ärzte und anderer Berufsgruppen.

Kürzlich meldete sich eine Psychotherapeutin. Sie arbeitet in einer Organisation, die viele Migranten behandelt. «Gestern war eine Patientin bei mir, die seit mehreren Sitzungen über eine ihrer Töchter spricht, die einen in ihrer Kultur inakzeptablen Fehler begangen hat. Nun hätten Mann und Sohn die Absicht, sie zu töten. Die Mutter sagt, dass eine reale Gefahr bestehe, wird dann unsicher, ist sich dann aber wieder sicher. Ich weiss nicht, was ich davon halten soll.» Eine immer heikle und schwierige Situation.

Zunächst ist zu bedenken, dass in dringenden Fällen, wenn ein schutzwürdiges Gut wie Gesundheit oder Leben in Gefahr ist, gesetzliche Bestimmungen wie die ärztliche Schweigepflicht in den Hintergrund treten. Mit anderen Worten - zunächst ist zu retten, was zu retten ist, danach regelt man die rechtlichen Fragen - und lässt sich eventuell von der Schweigepflicht entbinden. Meiner Auffassung nach kann (aus rechtlicher Sicht) und muss (aus ethischer Sicht) der/die Therapeut(in) die bedrohte(n) Person(en) und ggf. die Behörden in Kenntnis setzen, ob Polizei oder Staatsanwalt oder bei Minderjährigen zuständige kantonale Stellen. Notabene: In mehreren Kantonen sind Fachpersonen gesetzlich verpflichtet, bedrohte Minderjährige zu melden. Damit sind sie «automatisch» von ihrer Schweigepflicht entbunden.

Die Möglichkeit zur Meldung ist daher eindeutig vorhanden und sollte rechtzeitig genutzt werden. Im genannten Fall ist eine Besonderheit zu beachten. Es handelt sich um eine besorgte Mutter, und der Therapeutin ist die Tochter nicht bekannt. Weiss diese, dass sie in Gefahr ist? Es sollte also dafür gesorgt werden, dass sie gewarnt wird. So könnte sie selbst entscheiden, ob sie weggehen oder in einem Frauenhaus Schutz suchen will.

Denn es ist zu bedenken, dass bei einem Einschreiten der Behörden die Mutter als «Denunziantin» ihrerseits vom Umfeld bestraft werden könnte. Ein Grundsatz ist in allen Fällen, auch die Interessen und Risiken Dritter im Blick zu haben. Auch gegen den Willen der Mutter bzw. Tochter ist es möglich, den Fall zu melden. Allerdings sollte man zunächst versuchen, diese davon zu überzeugen, dass es richtig ist, so zu handeln - ohne die Dringlichkeit zu vergessen.

Was noch? Gibt es die Chance, die angespannte Lage in der Familie zu entschärfen, bevor öffentliche Stellen eingeschaltet werden? Gibt es innerhalb der kulturellen Gruppe Personen mit informeller Autorität, die diese Männer zur Raison bringen können? Es geht hier um eine Familie mit ausländischen Wurzeln. Hat sie möglicherweise Freunde «von hier», die einen Dialog (oder «Plurilog») initiieren und daran erinnern könnten, dass bestimmte Überzeugungen aus einer anderen Kultur hier nicht hinnehmbar - und auch strafbar sind? Besonders schwierig ist die Situation, wenn die Familie isoliert lebt.

Handelt es sich um einen jungen Menschen, kann man, möglichst mit seiner Zustimmung, zu Personen in seinem Umfeld, wie Gesundheitsfachpersonen, Sozialarbeitern, Erziehern, Lehrern oder Sporttrainern, Kontakt aufnehmen und sie nach ihrer Einschätzung fragen. Kollegen oder Arbeitgeber können manchmal hilfreiche Ratschläge geben.

Man kann auf transkulturelle Fragen spezialisierte Beratungsstellen konsultieren. Allgemein ist es in bestimmten Fragen wichtig, Meinungen von Experten für Gefahreinschätzung einzuholen. Oder ziehen Sie in Spitälern eingerichtete Abteilungen für Gewaltmedizin zu Rate. Denken Sie daran, dass man zusammen mit anderen oft klügere Einsichten hat als allein. Zudem können Sie jeden um Rat fragen, der Ihnen kompetent oder vertrauenswürdig erscheint, sofern Sie keine persönlichen Angaben preisgeben!

Noch eine letzte Bemerkung: Zwar erscheint es besser, zu früh vorzubeugen als zu spät. Aber je nach kultureller Gruppekönnen Massnahmen von offizieller Seite wie eine Bombe wirken und die Gefahr bergen, innerhalb der Familie weitere Gewalt und Chaos zu stiften. Deshalb sollte einerseits alles unternommen werden, was vor einem Eingreifen von aussen getan werden kann. Anderseits ist aber abzuschätzen, wie hoch die Gefahr ist, dass die Katastrophe eintritt, während man selbst noch auf die richtige Gelegenheit wartet ... Die (schwierige) Frage ist: Wie wendet man in dem Fall den Grundsatz Primum nil nocere richtig an?

Grundsätzlich sollte gelten: Wenn Gefahr im Verzuge ist, schützen! Wenn irgend möglich sollte die bedrohte Person den Massnahmen zustimmen.

Letztendlich nach Erfahrung, Herzen und Gewissen entscheiden.

Jean Martin, Mitglied der Redaktion und ehemaliger Kantonsarzt 